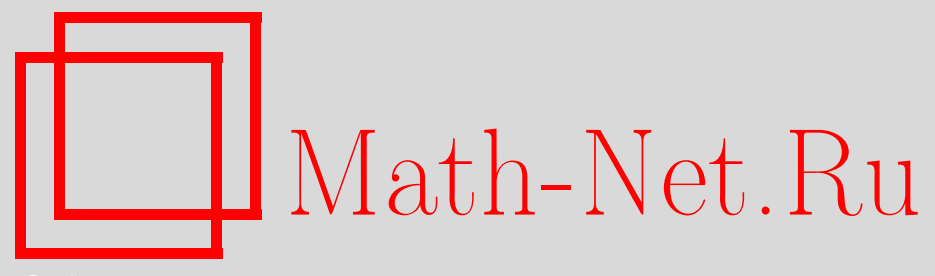

В. П. Чистяков, Статистики хи-квадрат, построенные по частотам неполных цепочек исходов независимых испытаний, Дискрет. матем., 2001, том 13, выпуск 4, 52-59

DOI: https://doi.org/10.4213/dm301

Использование Общероссийского математического портала Math-Net.Ru подразумевает, что вы прочитали и согласны с пользовательским соглашением http://www . mathnet.ru/rus/agreement

Параметры загрузки:

IP : 54.80 .73 .141

26 апреля 2023 г., 15:20:36 


\title{
Статистики хи-квадрат, построенные по частотам неполных цепочек исходов независимых испытаний
}

\author{
(C) 2001 г. В. П. Чистяков
}

\begin{abstract}
Выявление зависимостей в случайных последовательностях, когда зависимость проявляется на частотах длинных цепочек исходов, оказывается затруднительной из-за ограниченности возможностей вычислительной техники. В таких случаях приходится использовать различные критерии, определяемые статистиками, которые зависят от длинных цепочек подряд идущих знаков исследуемых последовательностей или от знаков, значительно удаленных друг от друга. Критерии такого типа можно найти в известных книгах Д. Кнута. В данной работе получены предельные распределения $\chi^{2}$-статистик, построенных по несплошным цепочкам исходов независимых испытаний, когда число испытаний стремится к бесконечности, исходы испытаний равновероятны, а их число фиксировано.

Работа выполнена при поддержке Российского фонда фундаментальных исследований, проект 99-01-00012, и Совете при президенте РФ по грантам и государственной поддержке ведущих научных школ, проект 00-15-96136.
\end{abstract}

\section{1. Определения и обозначения}

Пусть

$$
x_{1}, x_{2}, \ldots
$$

- последовательность независимых одинаково распределенных случайных величин, каждая из которых равномерно распределена на множестве $\{1,2, \ldots, N\}$. Рассмотрим последовательность

$$
\left(x_{t+r_{1}-1}, x_{t+r_{2}-1}, \ldots, x_{t+r_{v}-1}\right), \quad t=1,2, \ldots,
$$

$v$-цепочек, $1 \leqslant r_{1}<r_{2}<\ldots<r_{v}, v \geqslant 2$. Сплошная $s$-цепочка

$$
\left(x_{t}, x_{t+1}, \ldots, x_{t+s-1}\right)
$$

является наименьшей $s$-цепочкой, содержащей $t$-ю несплошную цепочку из (2), когда $r_{1}=1$ и $r_{v}=s$. Далее всегда предполагается, что $r_{1}=1$ и $r_{v}=s$. Положим

$$
C=\left\{r_{1}, r_{2}, \ldots, r_{v}\right\}, \quad \bar{C}=\{1,2, \ldots, s\} \backslash C .
$$


Обозначим $\nu(n, I)$ частоту (число появлений) $s$-цепочки $I=\left(i_{1}, i_{2}, \ldots, i_{s}\right)$ среди $s$-цепочек (3) при $t=1,2, \ldots, n$. Частоту цепочки $I^{C}=\left(i_{r_{1}}, i_{r_{2}}, \ldots, i_{r_{v}}\right)$ в $n$ первых членах последовательности (2) обозначим $\nu^{C}\left(n, I^{C}\right)$. Очевидно, что

$$
\nu^{C}\left(n, I^{C}\right)=\sum_{l \in \bar{C}} \sum_{i_{l}=1}^{N} \nu(n, I)
$$

Нормированные и центрированные частоты обозначим

$$
\xi^{C}\left(n, I^{C}\right)=\frac{\nu^{C}\left(n, I^{C}\right)-n / N^{v}}{\sqrt{n / N^{v}}}, \quad \xi\left(n, I^{C}\right)=\frac{\nu(n, I)-n / N^{s}}{\sqrt{n / N^{s}}}
$$

Вид статистики, имеющей в пределе $\chi^{2}$-распределение, оказался таким же, как и для сплошных цепочек, именно,

$$
X(n)=\sum_{I^{C}}\left(\xi^{C}\left(n, I^{C}\right)\right)^{2}-\sum_{\tilde{I}^{C}}\left(\tilde{\xi}^{C}\left(n, \tilde{I}^{C}\right)\right)^{2},
$$

где

$$
\begin{aligned}
I^{C} & =\left(i_{r_{1}}, i_{r_{2}}, \ldots, i_{r_{v}}\right), \\
\tilde{I}^{C} & =\left(i_{r_{1}}, i_{r_{2}}, \ldots, i_{r_{v-1}}\right), \\
\tilde{\xi}^{C}\left(n, I^{C}\right) & =\left(\tilde{\nu}^{C}\left(n, \tilde{I}^{C}\right)-n / N^{v-1}\right) / \sqrt{n / N^{v-1}}, \\
\tilde{\nu}^{C}\left(n . \tilde{I}^{C}\right) & =\sum_{i_{r_{v}}=1}^{N} \nu^{C}\left(n, I^{C}\right) .
\end{aligned}
$$

\section{2. Ковариации нормированных частот}

Часто используемые суммы

$$
\sum_{l \in C} \sum_{i_{l}=1}^{N}, \quad \sum_{l \in \bar{C}} \sum_{i_{l}=1}^{N}
$$

будем обозначать соответственно $\Sigma_{I}^{C}, \Sigma_{I}^{\bar{C}}$. Нетрудно проверить, что

$$
\xi^{C}(n, I)=\frac{1}{\sqrt{N^{s-v}}} \Sigma_{I}^{C} \xi(n, I)
$$

Отсюда получаем выражение для ковариаций

$$
\operatorname{cov}\left(\xi^{C}\left(n, I^{C}\right), \xi^{C}\left(n, J^{C}\right)\right)=\frac{1}{N^{s-v}} \Sigma_{I}^{\bar{C}} \Sigma_{J}^{\bar{C}} \operatorname{cov}(\xi(n, I), \xi(n, J))
$$

где $J=\left(j_{1}, \ldots, j_{s}\right)$. Известно (см. [2]), что при $n \rightarrow \infty$ существует предел

$$
\lim _{n \rightarrow \infty} \operatorname{cov}(\xi(n, I), \xi(n, J))=\sigma(I, J),
$$

где

$$
\begin{aligned}
& \sigma(I, J)=\delta(I, J)-\frac{2 s-1}{N^{s}}+\sum_{m=1}^{s-1} \frac{1}{N^{m}}\left(\delta_{i_{m+1} j_{1}} \ldots \delta_{i_{s} j_{s-m}}+\delta_{j_{m+1} i_{1}} \ldots \delta_{j_{s} i_{s-m}}\right), \\
& \delta(I, J)=\delta_{i_{1} j_{1}} \ldots \delta_{i_{s} j_{s}}
\end{aligned}
$$


и $\delta_{i j}$ - символ Кронекера.

Из (7) и (8) получаем, что существует предел ковариаций частот несплошных цепочек, равный

$$
\lim _{n \rightarrow \infty} \operatorname{cov}\left(\xi^{C}\left(n, I^{C}\right), \xi\left(n, J^{C}\right)\right)=\sigma^{C}\left(I^{C}, J^{C}\right)
$$

где

$$
\sigma^{C}\left(I^{C}, J^{C}\right)=\frac{1}{N^{s-v}} \Sigma_{I}^{\bar{C}} \Sigma_{J}^{\bar{C}} \sigma(I, J)
$$

При суммировании в (9) символов Кронекера $\delta_{i_{k} j_{l}}$, входящих в (8), возможны три случая, именно, $k, l \in C, k, l$ принадлежат разным множествам и $k, l \in \bar{C}$. В первом случае множитель $\delta_{i_{k} j_{l}}$ выносится за знак суммы. Во втором случае, например, если $k \in C, l \in \bar{C}$, множители $\delta_{i_{k} j_{l}}$ суммируются только по $l_{l}=1, \ldots, N$ и, следовательно,

$$
\sum_{l_{l}=1}^{N} \delta_{i_{k} j_{l}}=1
$$

Если же $k, l \in \bar{C}$, то суммирование проводится по двум индексам и

$$
\sum_{i_{k}, j_{k}=1}^{N}=N
$$

Отметим еще, что при суммировании произведений $\delta_{i_{m+1} j_{1}} \ldots \delta_{i_{s} j_{s-m}}$ из $m$-го слагаемого в (8), нужно суммировать по всем индексам $i_{k}$ и $j_{l}$ таким, что $k, l \in \bar{C}$, при этом $i_{k}$ и $j_{l}$ могут не входить в множества $\left(i_{m+1}, \ldots, i_{s}\right),\left(j_{1}, \ldots, j_{s-m}\right)$. Произведения $\delta_{j_{m+1} i_{1}} \ldots \delta_{j_{s} i_{s-m}}$ суммируются аналогично.

Удобно ввести обозначения

$$
\begin{aligned}
\gamma_{k} & = \begin{cases}1, & \text { если } k \in C, \\
0, & \text { если } k \in \bar{C},\end{cases} \\
D_{k l}^{(1)} & = \begin{cases}1, & \text { если } k, l \in C, \\
0 & \text { в остальных случаях, }\end{cases} \\
D_{k l}^{(2)} & = \begin{cases}1, & \text { если } k \in C, l \in \bar{C} \text { или } k \in \bar{C}, l \in C, \\
0 & \text { в остальных случаях, }\end{cases} \\
D_{k l}^{(3)} & = \begin{cases}1, & \text { если } k, l \in \bar{C}, \\
0 & \text { в остальных случаях. }\end{cases}
\end{aligned}
$$

Отметим, что

$$
\begin{aligned}
& D_{k l}^{(1)}=\gamma_{k} \gamma_{l}, \\
& D_{k l}^{(2)}=\left(\gamma_{k}-\gamma_{l}\right)^{2}, \\
& D_{k l}^{(3)}=\left(1-\gamma_{k}\right)\left(1-\gamma_{l}\right)
\end{aligned}
$$


Используя эти замечания, сумму частей $m$-го слагаемого в (9) представим в виде

$$
\begin{aligned}
S_{m}^{(I)} & =\Sigma_{i}^{\bar{C}} \Sigma_{J}^{(\bar{C}}\left(\prod_{k=1}^{s-m} \delta_{i_{m+k} j_{k}}\right) \\
& =\Pi_{m}^{C} \Pi_{m}^{\bar{C}} N^{\left(1-\gamma_{s-m+1}\right)+\left(1-\gamma_{s-m+2}\right)+\ldots+\left(1-\gamma_{s}\right)} N^{\left(1-\gamma_{1}\right)+\left(1-\gamma_{2}\right)+\ldots+\left(1-\gamma_{m}\right)},
\end{aligned}
$$

где

$$
\begin{aligned}
& \Pi_{m}^{C}=\prod_{k \in C, k \leqslant s-m}\left(D_{k, m+k}^{(1)} \delta_{j_{k} i_{k+m}}+D_{k, m+k}^{(2)}+N D_{k, m+k}^{(3)}\right), \\
& \Pi_{m}^{\bar{C}}=\prod_{k \in \bar{C}, k \leqslant s-m}\left(D_{k, m+k}^{1)} \delta_{j_{k} i_{k+m}}+D_{k, m+k}^{(2)}+N D_{k, m+k}^{(3)}\right) .
\end{aligned}
$$

Здесь два множителя $N$ в указанных степенях появляются при суммировании по индексам $i_{k} \notin\left(i_{m 1}, \ldots, i_{s}\right), j_{l} \notin\left(j_{1}, \ldots, j_{s-m}\right)$.

Нетрудно проверить, что

$$
\begin{aligned}
\Pi_{m}^{C} & =\prod_{k \in C, k \leqslant s-m}\left(\gamma_{k+m} \delta_{j_{k} i_{k+m}}+\left(1-\gamma_{k+m}\right)=\prod_{l \in C, l-m \in C, l \geqslant m+1} \delta_{i_{l} j_{l-m}}\right. \\
\Pi_{m}^{\bar{C}} & =\prod_{k \in \bar{C}, k \leqslant s-m}\left(\gamma_{k+m}+N\left(1-\gamma_{k+m}\right)\right) \\
& =N^{\left(1-\gamma_{m+1}\right)\left(1-\gamma_{1}\right)+\left(1-\gamma_{m+2}\right)\left(1-\gamma_{2}\right)+\ldots+\left(1-\gamma_{s}\right)\left(1-\gamma_{s-m}\right)}
\end{aligned}
$$

так как при $k \in C$

$$
D_{k, m+k}^{(1)}=\gamma_{k+m}, \quad D_{k, m+k}^{(2)}=1-\gamma_{k+m}, \quad D_{k, m+k}^{(3)}=0
$$

и при $k \in \bar{C}$

$$
D_{k, m+k}^{(1)}=0, \quad D_{k, m+k}^{(2)}=\gamma_{k+m}, \quad D_{k, m+k}^{(3)}=1-\gamma_{k+m} .
$$

Отсюда и из равенств $\gamma_{1}+\ldots+\gamma_{s}=v$ и

$$
\sum_{k=s-m+1}^{s}\left(1-\gamma_{k}\right)+\sum_{k=1}^{m}\left(1-\gamma_{k}\right)+\sum_{k=1}^{s-m}\left(1-\gamma_{k+m}\right)\left(1-\gamma_{k}\right)=s+m-2 v+\sum_{k=1}^{s-m} \gamma_{k} \gamma_{k+m}
$$

получаем, что

$$
S_{m}^{(I)}=N^{s+m-2 v+\gamma_{1} \gamma_{m+1}+\ldots+\gamma_{s-m} \gamma_{s}} \prod_{l, l-m \in C, l \geqslant m+1} \delta_{i_{l} j_{l-m}}^{\gamma_{l} \gamma_{l-m}} .
$$

Выражение для второй суммы $S_{m}^{(J)}$, соответствующей оставшейся части $m$-го слагаемого, получается из формулы для $S_{m}^{(I)}$, если поменять местами $i$ и $j$.

Непосредственно проверяется, что

$$
\begin{aligned}
\Sigma_{I}^{\bar{C}} \Sigma_{J}^{\bar{C}} \delta(I, J) & =N^{s-v} \prod_{l \in C} \delta_{i_{l} j_{l}}, \\
\Sigma_{I}^{\bar{C}} \sigma_{J}^{\bar{C}} 1 & =N^{2(s-v)} .
\end{aligned}
$$

Из формул (8)-(11) получаем следующее утверждение. 
Теорема 1. При $n \rightarrow \infty$ существуют предель

$$
\operatorname{cov}\left(\xi^{C}\left(n, I^{C}\right), \xi^{C}\left(n, J^{C}\right) \rightarrow \sigma^{C}\left(I^{C}, J^{C}\right),\right.
$$

əде

$$
\begin{aligned}
\sigma^{C}\left(I^{C}, J^{C}\right) & =\delta\left(I^{C}, J^{C}\right)-\frac{2 s-1}{N^{v}}+\sum_{m=1}^{s-1}\left(\prod_{l=m+1}^{s} \delta_{i_{l} j_{l-m}}^{\gamma_{l} \gamma_{l-m}}+\prod_{l=m+1}^{s} \delta_{j_{l} i_{l-m}}^{\gamma_{l} \gamma_{l-m}}\right) f_{m}, \\
\delta\left(I^{C}, J^{C}\right) & =\prod_{l \in C} \delta_{i_{l} j_{l}}, \\
f_{m} & =N^{-v+\gamma_{1} \gamma_{m+1}+\ldots+\gamma_{s-m} \gamma_{s}},
\end{aligned}
$$

$\gamma_{k}=1$ при $k \in C, \gamma_{k}=0$ при $k \in \bar{C}$, и произведения равны единиче, если множество индексов сомножителей пусто.

Применим полученный результат к случаю, когда

$$
v=3, \quad C=\left(r_{1}, r_{2}, r_{3}\right), \quad r_{1}=1, \quad r_{2}=r, \quad r_{3}-s .
$$

Если $r \neq(s+1) / 2$, то непустые произведения в (12) имеются только при $m=$ $r-1, s-r, s-1$ и равны соответственно $\delta_{i_{r} j_{1}}, \delta_{i_{s} j_{r}}, \delta_{i_{s} j_{1}} \delta_{j_{r} i_{1}}, \delta_{j_{s} i_{r}}, \delta_{j_{s} i_{1}}$. В этих случаях

$$
\sum_{k=1}^{s-m} \gamma_{k+m} \gamma_{k}=1
$$

в остальных случаях эта сумма равна нулю. Таким образом, сумма по $m$ в (12) равна

$$
\frac{1}{N^{2}}\left(\delta_{i_{r} j_{1}}+\delta_{i_{s} j_{r}}+\delta_{i_{s} j_{1}}+\delta_{j_{r} i_{1}}+\delta_{j_{s} i_{r}}+\delta_{j_{s} i_{1}}\right)+\sum_{m \neq r-1, s-r, s-1} \frac{2}{N^{3}}
$$

Отсюда и из (12) получаем следующее утверждение.

Следствие 1. В случае, когда

$$
C=(1, r, s), \quad 1<r<s, \quad I^{C}=\left(i_{1}, i_{r}, i_{s}\right), \quad J^{C}=\left(j_{1}, j_{r}, j_{s}\right), \quad 2 r \neq s+1,
$$

предельная ковариация $\sigma^{C}\left(I^{C}, J^{C}\right)$ определяется формулой

$$
\sigma^{C}\left(I^{C}, J^{C}\right)=\delta_{i_{1} j_{1}} \delta_{i_{r} j_{r}} \delta_{i_{s} j_{s}}-\frac{7}{N^{3}}+\frac{1}{N^{2}}\left(\delta_{i_{r} j_{1}}+\delta_{i_{s} j_{r}}+\delta_{i_{s} j_{1}}+\delta_{j_{r} i_{1}}+\delta_{j_{s} i_{r}}+\delta_{j_{s} i_{1}}\right)
$$

Если $2 r=s=1$, то три знака последовательности (1) берутся через одинаковые промежутки. В этом случае получается формула, совпадающая с формулой для сплошной $s$-цепочки при $s=3$.

Следствие 2. Если

$$
C=(1, r, s), \quad 1<r<s, \quad I^{C}=\left(i_{1}, i_{r}, i_{s}\right), \quad J^{C}=\left(j_{1}, j_{r}, j_{s}\right), \quad 2 r=s+1,
$$

mo

$$
\sigma^{C}\left(I^{C}, J^{C}\right)=\delta_{i_{1} j_{1}}+\delta_{i_{r} j_{r}}+\delta_{i_{s} j_{s}}-\frac{5}{N^{3}}=\frac{1}{N}\left(\delta_{i_{r} j_{1}} \delta_{i_{s} j_{r}} \delta_{j_{s} i_{r}}\right)+\frac{1}{N^{2}}\left(\delta_{i_{s} j_{1}}+\delta_{j_{s} i_{1}}\right)
$$




\section{3. Предельные распределения}

При $n \rightarrow \infty$ вектор частот $\left\{\nu^{C}\left(n, I^{C}\right)\right\}$ асимптотически нормален с нулевым вектором математических ожиданий и ковариационной матрицей $B=\left(\sigma^{C}\left(I^{C}, J^{C}\right)\right)$, где $\sigma^{C}\left(I^{C}, J^{C}\right)$ определено равенством (12). Предельное распределение квадратичной формы (6) от нормированных частот $\left\{\xi^{C}\left(n, I^{C}\right)\right\}$ совпадает с распределением этой квадратичной формы, если в ней заменить $\left\{\xi^{C}\left(n, I^{C}\right\}\right.$ на нормально распределенный вектор $\left\{\xi^{C}\left(I^{C}\right)\right\}$, соответствующий предельному распределению.

Заменим в (6) нормированные частоты $\tilde{\xi}^{C}\left(n, I^{C}\right)$ по формуле

$$
\tilde{\xi}^{C}\left(n, I^{C}\right)=\frac{1}{\sqrt{N}} \sum_{i_{r_{v}}=1}^{N} \xi^{C}\left(n, I^{C}\right) .
$$

Величину $X(n)$ можно представить в виде

$$
X(n)=\sum_{I^{C}} \sum_{J^{C}} A\left(I^{C}, J^{C}\right) \xi^{C}\left(n, I^{C}\right) \xi^{C}\left(n, J^{C}\right),
$$

где

$$
A\left(I^{C}, J^{C}\right)=\delta_{i_{r_{1}}} \ldots \delta_{i_{r_{v-1}}} j_{r_{v-1}}\left(\delta_{i_{r_{v}} j_{r_{v}}}-1 / N\right)
$$

Положим

$$
X=\sum_{I^{C}} \sum_{J^{C}} A\left(I^{C}, J^{C}\right) \xi^{C}\left(I^{C}\right) \xi^{C}\left(J^{C}\right) .
$$

Квадратичная форма $X$ имеет хи-квадрат распределение (см. [3, 4]), если

$$
A B A=A,
$$

где $A$ - матрица коэффициентов (15). Обозначим $(i, j)$-й элемент матрицы $D$ через $(D)_{i j}$. Проверим условие (16). Представим $N \times N$ ковариационную матрицу $B$ с элементами (12) в виде

$$
B=E+\frac{2 s-1}{N^{v}} F+\sum_{m=1}^{s-1}\left(G_{1, m}+G_{2, m}\right),
$$

где $N^{v} \times N^{v}$ матрицы $E, F, G_{1, m}$ и $G_{2, m}$ определяются заданием их элементов следующим образом:

$$
\begin{aligned}
(E)_{I^{C}, J C} & =\delta_{i_{r_{1}} j_{r_{1}}} \delta_{i_{r_{2}} j_{r_{2}}} \ldots \delta_{i_{r_{v}} j_{r_{v}}}, \\
(F)_{I^{C}, J^{C}} & =1, \\
\left(G_{1, m}\right)_{I^{C}, J^{C}} & =\prod_{r_{k}: m+1 \leqslant r_{k} \leqslant r_{v}} \delta_{i_{r_{k}}} j_{r_{k}-m}^{\gamma_{r_{k}-m}}, \\
\left(G_{2, m}\right)_{I^{C}, J^{C}} & =\prod_{r_{k}: m+1 \leqslant r_{k} \leqslant r_{v}} \delta_{i_{r_{k}}-m} j_{r_{k}}^{\gamma_{r_{k}-m}},
\end{aligned}
$$

Матрицу $A$ коэффициентов (15) квадратичной формы $X$ представим в виде

$$
A=E-\frac{1}{N} \tilde{E},
$$


где

$$
(\tilde{E})_{I^{C}, J^{C}}=\delta_{i_{r_{1}} j_{r_{1}}} \ldots \delta_{i_{r_{v-1}} j_{r_{v-1}}}
$$

При проверке условия (16) появятся матрицы $\tilde{G}_{1, m}$ и $\tilde{G}_{2, m}$ с элементами

$$
\begin{aligned}
& \left(\tilde{G}_{1, m}\right)_{I^{C}, J^{C}}=\prod_{r_{k}: m+1 \leqslant r_{k} \leqslant r_{v-1}} \delta_{i_{r_{k}}} j_{r_{k}-m}^{\gamma_{r_{k}-m}}, \\
& \left(\tilde{G}_{2, m}\right)_{I^{C}, J^{C}}=\prod_{r_{k}: m+1 \leqslant r_{k} \leqslant r_{v-1}} \delta_{i_{r_{k}}-m} j_{r_{k}}^{\gamma_{r_{k}-m}} .
\end{aligned}
$$

Заметим, что при $\gamma_{r_{v}-m}=0$ матрицы (21) совпадают с матрицами (18). При умножении матриц $A$ на $B$ и $A B$ на $A$, будут использоваться равенства

$$
\begin{array}{rlrl}
\tilde{E} F & =N F, \quad F \tilde{E}=N F, & \tilde{E}^{2}=N E, \\
\tilde{G}_{1, m}=N^{1-\gamma_{r_{v}-m}} \tilde{G}_{1, m}, & G_{1, m} \tilde{E}=N G_{1, m}, \\
\tilde{G}_{1, m} \tilde{E}=N \tilde{G}_{1, m}, & \tilde{E} G_{2, m}=G_{2, m} .
\end{array}
$$

Эти равенства проверяются непосредственно с использованием определения произведения матриц. Найдем, например, $\tilde{E} G_{1, m}$. Положим

$$
L^{C}=\left(l_{r_{1}}, \ldots, l_{r_{v}}\right)
$$

Тогда

$$
\begin{aligned}
\left(\tilde{E} G_{1, m}\right)_{I^{C}, J^{C}} & =\sum_{l_{r_{1}}, \ldots, l_{r_{v}}=1}^{N}(\tilde{E})_{I^{C}, J^{C}}\left(G_{1, m}\right)_{L^{C}, J^{C}} \\
& =\sum_{l_{r_{1}}, \ldots, l_{r_{v}}=1}\left(\prod_{\left\{r_{k}: r_{1} \leqslant r_{k} \leqslant r_{v-1}\right\}} \delta_{i_{r_{k}} l_{r_{k}}}\right)\left(\prod_{m+1 \leqslant r_{k} \leqslant r_{v-1}} \delta_{l_{r_{k}} j_{r_{k}}}^{\gamma_{r_{k}-m}}\right) \delta_{l_{r_{v}} j_{r_{v}}}^{\gamma_{r_{v}-m}} .
\end{aligned}
$$

Отсюда, учитывая равенства

$$
\begin{aligned}
\sum_{l_{r_{v}}=1}^{N} \delta_{l_{r_{v}} j_{r_{v}}}^{\gamma_{r_{v}-m}} & =N^{1-\gamma_{r_{v}-m}} \\
\sum_{l_{r_{k}}=1}^{N} \delta_{i_{r_{k}} l_{r_{k}}} \delta_{l_{r_{k} j_{r_{k}}}}^{\gamma_{r_{r_{k}-m}}} & =\delta_{i_{r_{k} j_{r_{k}}}}^{\gamma_{r_{k}-m}}
\end{aligned}
$$

получаем четвертое равенство в (22).

Умножим слева (17) на (19). Используя (22) и учитывая, что $E$ - единичная матрица, получим, что

$$
\begin{aligned}
A B= & E+\frac{2 s-1}{N^{v}} F+\sum_{m=1}^{s-1} f_{m}\left(G_{1, m}+G_{2, m}\right) \\
& -\frac{1}{N} \tilde{E}-\frac{2 s-1}{N^{v}} F-\frac{1}{N} \sum_{m=1}^{s-1} f_{m}\left(N^{1-\gamma_{r_{v}-m}} \tilde{G}_{1, m}+N G_{2, m}\right) \\
= & A+\sum_{\left\{m: \gamma_{r_{v}-m}=0\right\}} f_{m}\left(G_{1, m}-\tilde{G}_{1, m}\right)+\sum_{\left\{m: \gamma_{r_{v}-m}=0\right\}} f_{m}\left(G_{1, m}-\frac{1}{N} \tilde{G}_{1, m}\right) .
\end{aligned}
$$


Отсюда,

$$
A B=A+\sum_{\left\{m: \gamma_{r_{v}-m}=1\right\}} f_{m}\left(G_{1, m}-\frac{1}{N} \tilde{G}_{1, m}\right)
$$

так как $G_{1, m}=\tilde{G}_{1, m}$ при $\gamma_{r_{v}-m}=0$. Умножая обе части этого равенства справа на $A$, получаем (16).

Число степеней свободы хи-квадрат распределения равно следу матрицы $A B$. Нетрудно проверить, что

$$
\sum_{I^{C}}(A)_{I^{C}, I^{C}}=N^{v}-N^{v-1}
$$

и след матрицы $G_{1, m}-N^{-1} \tilde{G}_{1, m}$ при $\gamma_{r_{v}-m}=1$ равен нулю:

$$
\begin{aligned}
& \sum_{I^{C}}\left(\left(G_{1, m}\right)_{I^{C}, I^{C}}-\frac{1}{N}\left(\tilde{G}_{1, m}\right)_{I^{C}, I^{C}}\right) \\
& =\sum_{i_{r_{1}}, \ldots, i_{r_{v-1}}} \prod_{m+1 \leqslant r_{k} \leqslant r_{v-1}} \delta_{i_{r_{k}} i_{r_{k}-m}}^{\gamma_{r_{k}-m}} \sum_{i_{r_{v}}=1}^{N} \delta_{i_{r_{v}} i_{r_{v}-m}} \\
& -\frac{1}{N} \sum_{i_{r_{1}}, \ldots, i_{r_{v-1}}} \prod_{m+1 \leqslant r_{k} \leqslant r_{v-1}} \delta_{i_{r_{k}} i_{r_{k}-m}}^{\gamma_{r_{k}-m}}=0 .
\end{aligned}
$$

Отсюда и из (23) следует, что след $A B$ совпадает со следом $A$.

Таким образом, получена следующая теорема.

Теорема 2. При $n \rightarrow \infty$ распределение квадратичной формы $X(n)$, определенной равенством (6), сходитсл $к$ централъному хи-квадрат распределению $c N^{v}-N^{v-1}$ степенями свободи.

\section{Список литературы}

1. Кнут Д., Искусство программирования для ЭВМ. Получисленные алгоритмы. т. 2. Мир, Москва, 1977.

2. Селиванов Б. И., Чистяков В. П., Последовательный $\chi^{2}$-критерий, построенный по $s$-цепочкам состояний цепи Маркова. Дисхретная математиха (1977) 9, №4, 127-136.

3. Dik J. J., Gunst M. C. M., The distribution of general quadratic form in normal variables. Stat. Neerlandica (1985) 39, №1, 14-26.

4. Тихомирова М. И., Чистяков В. П., О статистиках типа $\chi^{2}$, построенных по выходу конечного автомата. Труды по дискретной математике (1998) 2, 305-314.

Статья поступила 02.07.2001. 